\title{
Few-Particle Vortex Cluster Equilibria in Bose-Einstein Condensates: Existence and Stability
}

\author{
Anna M. Barry ${ }^{1}$, PG Kevrekidis ${ }^{1,2}$ \\ ${ }^{1}$ Institute for Mathematics and its Applications, University of Minnesota, \\ Minneapolis, MN 55455 \\ 2 Department of Mathematics and Statistics, University of Massachusetts, \\ Amherst MA 01003-4515, USA
}

\begin{abstract}
Motivated by recent experimental and theoretical studies of fewparticle vortex clusters in Bose-Einstein condensates, we consider the ordinary differential equations of motion and systematically examine settings for up to $N=6$ vortices. We analyze the existence of corresponding stationary state configurations and also consider their spectral stability properties. We compare our particle model results with the predictions of the full partial differential equation system. Whenever possible, we propose generalizations of these results in the context of clusters of $N$ vortices. Some of these, we can theoretically establish, especially so for the $\mathrm{N}$-vortex polygons, while others we state as conjectures, e.g. for the $\mathrm{N}$-vortex line equilibrium.
\end{abstract}

\section{Introduction}

The realm of Bose-Einstein condensates (BECs) has offered in the past two decades a pristine setting for the exploration of numerous nonlinear wave structures and their interactions [1, 2, 3. One of the most prominent examples of such states that has received considerable attention consists of matter wave vortices, which have by now been reviewed in numerous works [4, 5, 6, 7, 8. Most of the relevant experimental studies have been concerned with various techniques of producing single charge vortices. Corresponding examples include, among others, phase imprinting methods between two hyperfine states [9, stirring the BEC above a critical rotation speed [10, supercritical dragging of defects through the BEC [11, 12, quenching through the condensation quantum phase transition [13, 14 or the effectively nonlinear interference of atomic BEC fragments [15]. In addition, experimental efforts were also focused on producing vortices of higher topological charge [16, as well as on providing large amounts of angular momentum with the aim of generating robust triangular vortex lattices [17.

On the other hand, considerably less experimental effort was originally invested on the exploration of clusters of few vortices. It was realized early that such clusters with same circulation vortices could be created [10] and the expectation was that such states in the presence of angular momentum would shape up into canonical polygons 18 with or without a vortex located at the center. However, in more recent experimental efforts, states with two vortices in the form of a counter-circulating vortex dipole 12, 14, 19, as well as vortex tripoles 20, (with two vortices of one sign, and one vortex of the opposite sign) have been produced and their dynamics monitored. A 
very recent work has also produced sets of 2-, 3-, 4-vortices exploring their dynamics in the absence of a rotational angular momentum induced term 21. These experimental works have, in turn, either had as a preamble 22, 23, 24, 25, 26, 27, 28, 29, 30. or subsequently motivated 31, 32, 33, 34, 35. studies on the statics, stability and dynamics of such vortex clusters (predominantly, in fact, the vortex dipole).

Most of the above theoretical works on the vortex clusters have stemmed from an improved understanding of the underlying partial differential equation (PDE) which describes the pancake-shaped (i.e., quasi-two-dimensional) condensates which contain such clusters. However, in parallel, a growing fraction of the literature has been advocating [19, 21, 30, 34, 35] the usefulness of a particle model corroborating the two principal features of the vortex motion. These are that each of the vortices has a precessional motion (dictated by its charge, distance from the origin of the parabolic trap confining the BEC and characteristics of the BEC, namely its background density at the center characterizing the so-called chemical potential $\mu$ and the trap confinement frequency $\Omega$ ) and also has a relative position dependent interaction with other vortices present in the BEC. In the limit of large chemical potentials (so-called Thomas-Fermi limit), where the size of the vortex core shrinks effectively to a point, hence the structure of the core plays no role in the dynamics, it is expected that incorporating these two principal features into particle-based ordinary differential equations (ODEs) should be sufficient to capture the possible vortex cluster equilibria and to assess their (spectral) stability. This approach can, in fact, also be justified rigorously; see for example the derivation of the relevant ODEs in [36].

The aim of the present paper is to carry out the above mentioned program to the extent that it is possible analytically and/or in a numerically assisted form. We focus especially on the context of configurations that arise as stationary states from the system with a few vortices i.e., $N=3$ up to $N=6$. We then attempt to generalize the conclusions drawn from these low dimensional systems to the extent possible to larger dimensional ones carrying, however, suitable symmetries. As principal examples, we present the case of $\mathrm{N}$-gons where the vortices occupy the vertices of a canonical polygon, as well as that where the vortices are aligned along an axis of the BEC. In the former, we can prove some of the relevant stability conclusions (either analytically or in a numerically assisted form), while in the latter, we conjecture the general result based on our numerical observations, but leave the relevant proof as an open problem for future study.

Our presentation is structured as follows. In section II, we briefly present the theoretical setup, equations of motion and associated conservation laws. In section III, we focus on the realm of small vortex numbers $N=3, \ldots 6$, while in section $\mathrm{IV}$, we attempt to generalize our conclusions to larger $N$, under suitable symmetry constraints. In section $\mathrm{V}$, we summarize our findings and present our conclusions, as well as identify a number of directions for future work.

\section{Theoretical Setup}

At the PDE level, the system of interest can be described by a two-dimensional equation of the Nonlinear Schrödinger (NLS) or Gross-Pitaevskii (GPE) type, where the trap strength is parametrized by an effective frequency $\Omega_{e f f}=\omega_{r} / \omega_{z}$ (i.e., the ratio between the in-plane and perpendicular to the plane trapping frequencies) and the density (at the center of the trap) by the chemical potential parameter $\mu$, directly associated with the number of atoms in the BEC. Details about the PDE level 
Few-Particle Vortex Cluster Equilibria in Bose-Einstein Condensates: Existence and Stability3

description and the reduction from the original three-dimensional (3d) system to the effective two-dimensional (2d) one can be found in 32 for our setup. In some sense, our work will naturally complement the above manuscript, as the latter considers how vortex cluster states emerge from the linear limit of the quantum harmonic $(2 \mathrm{~d})$ oscillator and bifurcations of nonlinear states from linear states thereof (i.e., regime of small chemical potential). Here, on the other hand, we will focus on the opposite limit of large chemical potential and the particle system emerging when considering the vortices as isolated precessing and interacting entities characterized by their position within the two-dimensional plane.

In this spirit, let $\mathbf{x}_{j}=\left(x_{j}, y_{j}\right)$ be a point vortex in the BEC system with charge $S_{j}$ for $j=1, \ldots, N$ and consider the system

$$
\begin{aligned}
& \dot{x}_{j}=-S_{j} \Omega y_{j}-\frac{b}{2} \sum_{k \neq j}^{N} S_{k} \frac{y_{j}-y_{k}}{\left|\mathbf{x}_{j}-\mathbf{x}_{k}\right|^{2}} \\
& \dot{y}_{j}=S_{j} \Omega x_{j}+\frac{b}{2} \sum_{k \neq j}^{N} S_{k} \frac{x_{j}-x_{k}}{\left|\mathbf{x}_{j}-\mathbf{x}_{k}\right|^{2}}
\end{aligned}
$$

where we fix $b=2$ for simplicity $\mathbf{t} . \Omega$ in Eqs. (11)-(2) is the precession frequency of a single vortex in a trap, which is well-known to depend on the effective trap frequency $\Omega_{\text {eff }}$ and the number of atoms in the BEC (as characterized by the so-called chemical potential) [14, 30.

The above system is Hamiltonian with

$$
H\left(x_{1}, y_{1}, \ldots, x_{N}, y_{N}\right)=-\Omega \sum_{j=1}^{N} S_{j} r_{j}^{2}+\sum_{j<k}^{N} S_{j} \log \left(r_{j k}^{2}\right)
$$

where $r_{j k}=\left|z_{j}-z_{k}\right|$, and $z_{j}$ is the complex variable $z_{j}=x_{j}+i y_{j}$. In terms of this variable, the equations reduce to

$$
i \dot{z}_{j}=-S_{j} \Omega z_{j}+\sum_{k \neq j}^{N} \frac{S_{k}}{\bar{z}_{j}-\bar{z}_{k}} .
$$

We also note in passing that that the angular momentum $L=\sum S_{j} r_{j}^{2}$ is also a conserved quantity for the system of vortices. We use the term angular momentum for this conservation law in line with the tradition stemming from the literature of point vortices in fluid mechanics [38, 39] (rather than the angular momentum of the full quantum mechanical problem). Finally, it will be useful to cast the system in polar coordinates $\left(r_{j}, \theta_{j}\right)$, in which case the equations of motion become:

$$
\begin{aligned}
\dot{r}_{j} & =\sum_{k \neq j} \frac{S_{k} r_{k} \sin \left(\theta_{k}-\theta_{j}\right)}{r_{j k}^{2}} \\
r_{j} \dot{\theta}_{j} & =r_{j} S_{j} \Omega+\sum_{k \neq j} \frac{S_{k}}{r_{j k}^{2}}\left(r_{j}-r_{k} \cos \left(\theta_{j}-\theta_{k}\right)\right) .
\end{aligned}
$$

$\ddagger$ Our considerations herein will not be significantly affected by the precise value of $b$, as long as the latter assumes a constant value proximal to the one of the homogeneous limit that we assume here. We should note, however, for completeness that to improve the agreement between the ODE as regards the precise location of the fixed points, the work of [30] and others thereafter, considered an effective value of $b=1.35$. This was intended to account for the density-induced "screening" effect associated with the vortex interaction. A first-principles accounting of such screening would necessitate the study of integro-differential equations as analyzed in [37. [see Eq. (21) therein]. 
Few-Particle Vortex Cluster Equilibria in Bose-Einstein Condensates: Existence and Stability4

It should be noted here that in the present work the precession frequency of a single vortex will be assumed to be constant, an assumption that is fairly accurate between the center of the trap confining the BEC and half of its radial extent (the latter is often referred to as the Thomas-Fermi radius). More general position dependent precession frequency expressions can be used also in connection to experiments, such as most notably $\tilde{\Omega}_{j}=\Omega /\left(1-r_{j}^{2}\right)[14$, 19, 21]. However we have checked that these do not modify the existence or stability conclusions for the solutions presented in this paper. Hence, for simplicy of the exposition, we restrict our presentation to the constant $\Omega$ case hereafter.

\section{Small $N$}

We now focus more specifically on the case of small vortex clusters. It will be clear from what follows that the cases of interest to us will be those where the vortices do not all carry the same charge. In the latter case, the rotation imposed by the precession is only enhanced by the rotation induced by the interaction and hence the vortices cannot find themselves in a situation of genuine equilibrium. Rather, in the latter case, one can only talk about rigidly rotating states as was examined in the recent work of 21 for $N=2,3$ and 4 . For large $N$, the latter setting has been examined too, with a recent example being the work of [40]; see also therein for relevant references. Here, on the other hand, we deal with genuine equilibria of the vortex cluster system and hence none of our configurations carry vortices of a single charge type.

\section{1. $N=3$}

The case $N=2$ was examined in detail in 19, 33, in which the dipole was found to be the only fixed point and is linearly stable. Hence, we start by focusing our considerations to the case of $N=3$.

Proposition 3.1. When $N=3$, the only fixed point of the particle system is a collinear configuration.

Proof. Without loss of generality, fix $y_{1}=0$. Suppose $\left(x_{1}, 0, x_{2}, y_{2}, x_{3}, y_{3}\right)$ with charges $S_{1}, S_{2}$ and $S_{3}$ is a fixed point of the system. Fix $S_{1}=1$. Under these assumptions, the equations read

$$
\begin{aligned}
& \frac{S_{2} y_{2}}{r_{12}^{2}}=\frac{-S_{3} y_{3}}{r_{13}^{2}} \\
& \frac{S_{3}\left(y_{2}-y_{3}\right)}{r_{23}^{2}}=-S_{2} \Omega y_{2}-\frac{y_{2}}{r_{12}^{2}} \\
& \frac{S_{2}\left(y_{3}-y_{2}\right)}{r_{23}^{2}}=-S_{3} \Omega y_{3}-\frac{y_{3}}{r_{13}^{2}}
\end{aligned}
$$

There are three possible cases to consider: $S_{2}=S_{3}= \pm 1, S_{2}=-S_{3}=1$. In each case, we obtain the relation $y_{2}=-y_{3}$. For instance, consider $S_{2}=-S_{3}=1$. Then,

$$
\begin{aligned}
& \frac{y_{2}}{r_{12}^{2}}=\frac{y_{3}}{r_{13}^{2}} \\
& \frac{y_{3}-y_{2}}{r_{23}^{2}}=-\Omega y_{2}-\frac{y_{2}}{r_{12}^{2}}
\end{aligned}
$$


Few-Particle Vortex Cluster Equilibria in Bose-Einstein Condensates: Existence and Stability5

$$
\frac{y_{3}-y_{2}}{r_{23}^{2}}=\Omega y_{3}-\frac{y_{3}}{r_{13}^{2}}
$$

Thus inserting the first relation into the second equation and equating the second and third equations yields $y_{2}=-y_{3}$.

Note that even though we have satisfied the equations for equilibrium in $\mathrm{x}$, it is still possible $\dot{\mathbf{y}} \neq 0$. To see that a true collinear configuration exists, fix $\Omega=1$ and set $S_{1}=1, S_{2}=S_{3}=-1$. One can check that the configuration $\mathbf{x}_{1}=(0,0), \mathbf{x}_{2}=\left(0, \frac{\sqrt{2}}{2}\right)$ and $\mathbf{x}_{3}=\left(0,-\frac{\sqrt{2}}{2}\right)$ satisfies the fixed point equations.

One can then check by a direct calculation that the collinear fixed point is linearly unstable. The eigenvalues of the linearization matrix are $\lambda_{1,2}= \pm i \sqrt{5}, \lambda_{3,4}= \pm \sqrt{7}$ and $\lambda_{5,6}=0$. In general we expect the collinear configuration to have $N-2$ real directions of instability. This is consonant with the conclusions of [30, 32.

\section{2. $N=4$}

The case $N=4$ is more subtle, as the system exhibits more than one fixed points, as we show analytically below. Unlike the classical point vortex problem, the system (1)-(2) does not exhibit translational symmetry and therefore the "center of vorticity" is not conserved. In fact,

$$
\begin{aligned}
& \sum_{j=1}^{N} S_{j} \dot{x}_{j}=-\Omega \sum_{j=1}^{N} y_{j} \\
& \sum_{j=1}^{N} S_{j} \dot{y}_{j}=\Omega \sum_{j=1}^{N} x_{j}
\end{aligned}
$$

which implies that the fixed points sought herein must have a center of mass located at $(0,0)$. Moreover, the system exhibits rotational symmetry which is clear by replacing $\theta \mapsto \theta+\omega t$. We make the following conjecture.

Conjecture 3.2. All fixed points of (1)-(圆) are symmetric about the origin.

Assuming the conjecture is true, one can prove

Proposition 3.3. For $N=4$, the only fixed points of (1)-(2) are square or collinear (i.e., one in which all the vortices are located on a line going through the origin) configurations.

Proof. Choosing $\mathbf{x}_{1}=\left(x_{1}, 0\right)$ implies that $\mathbf{x}_{2}=\left(-x_{1}, 0\right)$. Then (10)-(11) imply $x_{3}=-x_{4}$ and $y_{3}=-y_{4}$. If $y_{3}=0$, the configuration is collinear, so assume $y_{3} \neq 0$. Then the equation for $\dot{x}_{1}$ yields

$$
\frac{S_{3}}{r_{13}^{2}}=\frac{S_{4}}{r_{14}^{2}} \text {. }
$$

Thus $r_{13}=r_{14}$, but since the configuration is symmetric about the origin this implies that it is a square.

Fixing $\mathbf{x}_{1}=(1,0), \mathbf{x}_{2}=(-1,0), \mathbf{x}_{3}=(0,1), \mathbf{x}_{4}=(0,-1), S_{1}=S_{2}=1$, and $S_{3}=S_{4}=-1$ gives a square fixed point with $\Omega=\frac{1}{2}$. Linearizing about this equilibrium yields eigenvalues

$$
\lambda_{1,2}= \pm \frac{i}{\sqrt{2}}, \lambda_{3,4}=\lambda_{5,6}= \pm \frac{i}{4}, \lambda_{7,8}=0
$$


Few-Particle Vortex Cluster Equilibria in Bose-Einstein Condensates: Existence and Stability6

with corresponding eigenvectors

$$
\begin{aligned}
& v_{1}=\{1 / 3 i(i+2 \sqrt{2}), 1 / 3(1-2 i \sqrt{2}), 1 / 3(1-2 i \sqrt{2}), 1 / 3 i(i+2 \sqrt{2}),-1,-1,1,1\}=\bar{v}_{2} \\
& v_{3}=\{0, i, 0, i, 0,1,0,1\}=\bar{v}_{4} \\
& v_{5}=\{-i, 0,-i, 0,1,0,1,0\}=\bar{v}_{6} \\
& v_{7}=\{-1,-1,1,1,1,-1,-1,1\} \\
& v_{8}=\mathbf{0} .
\end{aligned}
$$
where

Fixing $\Omega=\frac{1}{2}$ gives a collinear fixed point $\mathbf{x}_{1}=(c, 0)=-\mathbf{x}_{3}, \mathbf{x}_{2}=(d, 0)=-\mathbf{x}_{4}$

$$
\begin{aligned}
c & =\sqrt{1+\sqrt{2(-1+\sqrt{2})}} \\
d & =\frac{1}{2}\left(10 \sqrt{1+\sqrt{2(-1+\sqrt{2}})}-9(1+\sqrt{2(-1+\sqrt{2})})^{3 / 2}\right. \\
& \left.+4(1+\sqrt{2(-1+\sqrt{2})})^{5 / 2}-(1+\sqrt{2(-1+\sqrt{2})})^{7 / 2}\right) .
\end{aligned}
$$

This configuration has eigenvalues $\lambda_{1,2} \approx \pm 2.15, \lambda_{3,4} \approx \pm 1.43 i, \lambda_{5,6} \approx \pm .914$, $\lambda_{7,8}=0$, and hence possesses $N-2=2$ directions of instability.

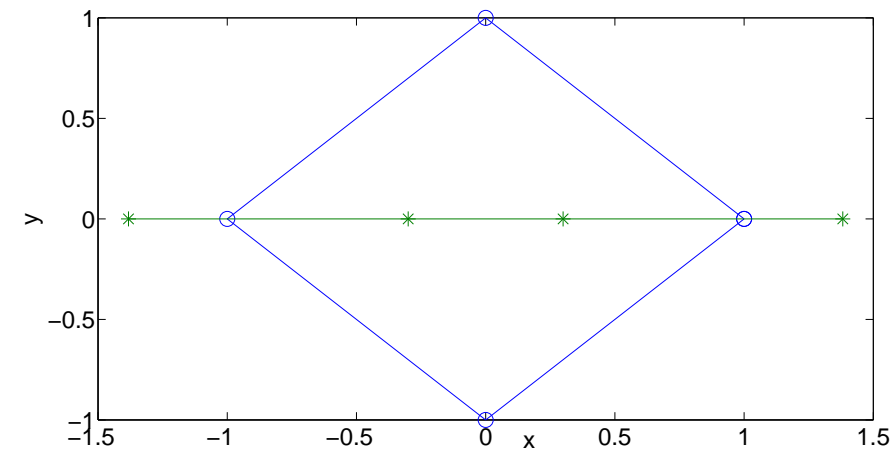

Figure 1: Fixed points of the system in the case of $N=4$ and with $\Omega=\frac{1}{2}$. The square configuration has the vortices denoted by circles, while the collinear one has them denoted by stars. Notice that adjacent vortices have opposite sign (unit magnitude) charge.

These results are consonant with the corresponding findings of the 2d PDE of the NLS/GPE type [30, 32. For the square configuration the PDE predicts that such a state exists even at the linear limit and bifurcates therefrom as a linearly stable equilibrium (see also [41]) with the exception of a possibility for an oscillatory instability (Hamiltonian Hopf bifurcation) which can arise for an intermediate range of values of $\mu$. Nevertheless, the state is linearly stable in the limit of large $\mu$. It should be highlighted that this state also has a neutral direction because of its radial symmetry within the isotropic two-dimensional parabolic trap. Such a direction is shared also by the collinear configuration which also has a pair of zero eigenvalues due to its invariance under rotations of its linear axis. Moreover, the latter configuration has 2 directions of instability, in line with the expectation that each higher collinear configuration will have an additional real eigenvalue pair (than the previous one - starting with the 
dipole that has none, then the tripole has one, the aligned quadrupole two, etc.). This expectation stems from the supercritical nature of the bifurcation of these collinear states from the dark soliton state as discussed in [30, 32].

\section{3. $N=5$}

In the next section we prove that for general $N$ odd the $N$-gon (i.e., the canonical polygon with the vortices at its vertices) is not a fixed point. We thus hereafter investigate other configurations, confining our considerations to ones that are symmetric about the origin. Fix $\left(x_{1}, y_{1}\right)=(0,0)$ and $\left(x_{2}, y_{2}\right)=\left(r_{1}, 0\right)$. Then by symmetry $\left(x_{3}, y_{3}\right)=\left(-r_{1}, 0\right)$. Set $\left(x_{4}, y_{4}\right)=\left(r_{2} \cos \theta_{2}, r_{2} \sin \theta_{2}\right), 0 \leq \theta_{2}<\pi$, so that $\left(x_{5}, y_{5}\right)=\left(-r_{2} \cos \theta_{2},-r_{2} \sin \theta_{2}\right)$. We compute

$$
\dot{x}_{1}=\frac{\left(S_{3}-S_{5}\right) \sin \theta_{2}}{r_{2}}
$$

and so either $\sin \theta_{2}=0$ or $S_{3}=S_{5}$. Assuming the latter holds we find from the equation for $y_{1}$ that $S_{2}=S_{4}$. Then from the equation for $x_{2}$ it follows that $\sin \left(2 \theta_{2}\right)=0$. If we choose the root $\theta_{2}=\frac{\pi}{2}$, then the configuration must be a square or rhombus centered on the origin. Here, two cases are physically relevant. One where the vortices alternate in sign in the counterclockwise direction with the central vortex being of either sign, and another where the outer vortices have the same sign opposite to the sign of the vortex at the origin. In the first case, we find that the system has no fixed points. In the second, we find that if the central vortex has charge $M>0$ and the outer vortices have charge -1 , then the system has a fixed point if and only if $r_{1}=r_{2}$. As an example, when the radius is one and $M=2$ we compute the configuration to be unstable with eigenvalues $\lambda_{1,2} \approx 3.14 i, \lambda_{3,4}=\sqrt{6}$, $\lambda_{5,6} \approx-1.15 \pm 1.07 i, \lambda_{7,8} \approx 1.15 \pm 1.07 i$ and $\lambda_{9,10}=0$. More generally, considering the algebraic constraints, one finds that the configuration with a square surrounding the central vortex can be realized provided suitable contraints connecting the central vortex charge, the surrounding charges, the precession frequency and the square's radius. The relevant condition reads

$$
r_{1}^{2}=\frac{2 M-3 \tilde{M}}{2 \Omega \tilde{M}}
$$

where $-\tilde{M}$ are the surrounding charges to the central one. It is thus clear that such configurations will only exist if $\tilde{M} \times(2 M-3 \tilde{M})>0$ and assuming $\tilde{M}>0$ without loss of generality, this leads to $M>3 \tilde{M} / 2$. In the case of $\tilde{M}=1$, the lowest charge that will work is $M=2$. This configuration can be found to be definitely unstable due to a real pair $\lambda= \pm 2 \tilde{M}^{3 / 2} \Omega \sqrt{4 M-2 \tilde{M}} /(2 M-3 \tilde{M})$, while other eigenvalues have more complicated functional forms not provided here. As in the above numerical example, we find a quartet of complex eigenvalues, a real pair, an imaginary pair and a neutral pair associated with the rotational invariance of such a 5 -vortex state. There are two observations to make here in connection to this. This configuration is the same as the one labeled " $5 \mathrm{x}$ " in the work of 32 \&. The second is that in line with the numerical observations of [32, we find that this configuration at large $\mu$ contains a complex eigenfrequency quartet, as well as a real eigenvalue pair as manifestations of its instability (see accordingly the 3rd row, right panel of Fig. 4 in p. 1453 of [32]).

$\S$ However, note that inadvertently the latter work mentioned a quadrupole as surrounding the central vortex in p. 1453; the correct statement is that 4 same charge vortices, opposite in sign to the doubly charged central one are surrounding it. 
We now turn to the case when $\theta_{2}=0$; here, we have a collinear fixed point which only exists when the vortices have alternating charges. This fixed point can be described by the configuration $(0,0),\left( \pm r_{1}, 0\right),\left( \pm r_{2}, 0\right)$ where

$$
\begin{aligned}
& r_{1}=\sqrt{\frac{2-\sqrt{3}}{2 \Omega}} \\
& r_{2}=\sqrt{\frac{\sqrt{3}}{2 \Omega}}
\end{aligned}
$$

This configuration was computed to be unstable with eigenvalues $\lambda_{1,2} \approx \pm 6.95 \Omega$, $\lambda_{3,4} \approx \pm 6.69 \Omega, \lambda_{5,6} \approx \pm 3.64 i \Omega, \lambda_{7,8} \approx \pm 2.8 \Omega, \lambda_{9,10}=0$ and so again we see that there are $N-2=3$ directions of real instability, as well as a neutral direction. This is in line with the expectations of the earlier works of 30, 32, examining the PDE limit of such 5-vortex collinear configurations.

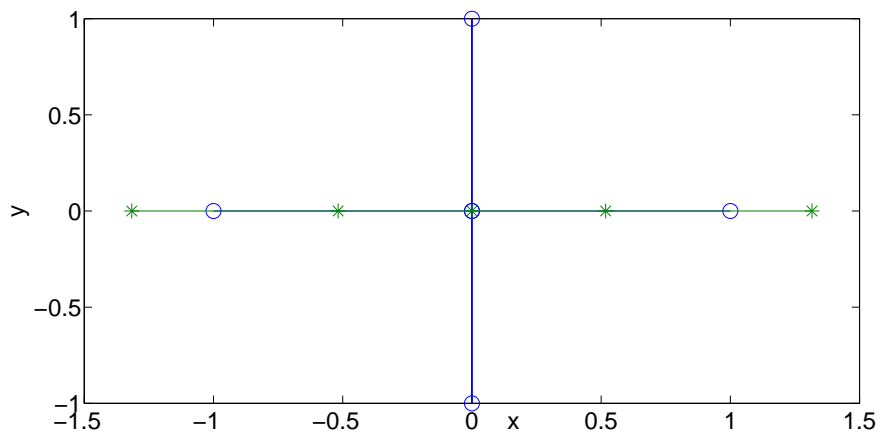

Figure 2: The prototypical fixed points that exist for $\Omega=\frac{1}{2}$ and $N=5$. The circles denote the example where an $M=2$ vortex at the origin is surrounded by 4 vortices of charge $-\tilde{M}=-1$ over the nodes of a square each at a unit distance from the origin. The stars denote the collinear configuration of alternating sign (unit magnitude) charges.

\section{4. $N=6$}

We are aware of two fixed points when $N=6$ : the hexagon and the collinear configuration. Both of these are configurations of alternating charges (either along the ring or along a line, respectively). The linearization about the hexagon when all vortices are on the unit circle and $\Omega=\frac{1}{2}$ yields the following eigenvalues

$$
\lambda_{1,2}= \pm \frac{3 i}{\sqrt{2}}, \lambda_{3,4}=\lambda_{5,6}= \pm i, \lambda_{7,8}=\lambda_{9,10}= \pm \frac{1}{\sqrt{2}}, \lambda_{11,12}=0
$$

and so, unlike the square, the hexagon is unstable. This is in line with earlier observations at the PDE level for this vortex ring, see e.g. the discussion of [32] (top right of Fig. 5 in p. 1454 and associated discussion). There, it is inferred (coming from the opposite limit of small chemical potential) that the hexagon supercritically bifurcates from the already unstable (even off of and near the linear limit) state of the dark soliton ring [2, inheriting its instability. 
The collinear configuration can be represented by $( \pm a, 0),( \pm b, 0),( \pm c, 0)$ where $a \approx 0.23, b \approx 0.67$ and $c \approx 1.58$ were computed numerically. The eigenvalues of the linearization about this fixed point were computed to be $\lambda_{1,2} \approx \pm 4.88, \lambda_{3,4} \approx \pm 4.86$, $\lambda_{5,6} \approx \pm 2.65, \lambda_{7,8} \approx \pm 2.2 i, \lambda_{9,10} \approx \pm 0.96$, and $\lambda_{11,12}=0$ and so this configuration also has $N-2=4$ directions of instability. We note in this case too that in order to generalize the relevant results in the case of arbitrary $\Omega$, one has to scale the fixed point positions by $1 / \sqrt{\Omega}$ and the corresponding eigenvalues linearly by $\Omega$.

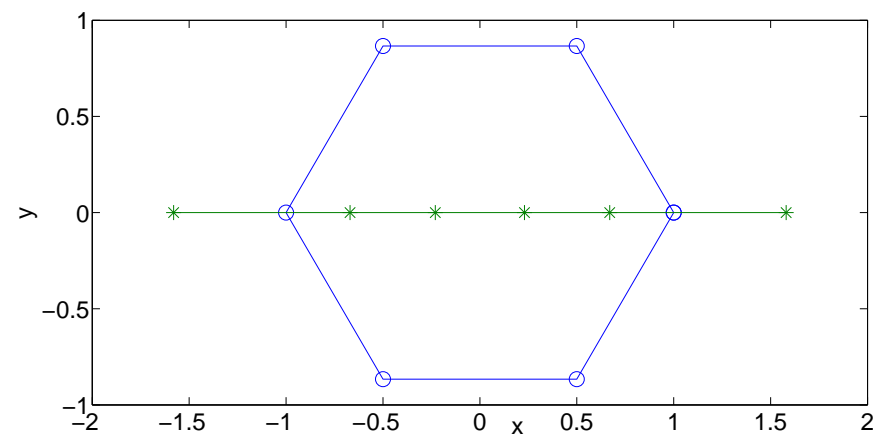

Figure 3: Two fixed points when $N=6$ and again for $\Omega=\frac{1}{2}$. The hexagonal configuration is shown by (blue) circles, while the collinear one by (green) stars. We again note as in the case of $N=4$ that adjacent vortices have opposite charges between each other.

\section{Large $N$}

\subsection{The $N$-gon fixed point}

Up to now, we have provided detailed (existence and stability) features of setups containing only small numbers of vortices. We now generalize our conclusions, by offering some proofs, as well as some conjectures for more general vortex configurations featuring large numbers of vortices $N$. As we will see, there are some significant differences between the cases of an even number of vortices and those of an odd number of vortices, both at the level of existence, as well as at that of stability. We will assume hereafter that the vortices are of alternating charge, namely $S_{j}=(-1)^{j+1}$, $j=1,2, \ldots, N$.

Proposition 4.1. The polygonal $N$-vortex (hereafter, sometimes referred to as " $N$ gon") configuration described above is a fixed point of the point vortex system if and only if $N$ is even. Moreover, the configuration is linearly stable for $N=4$ and linearly unstable otherwise.

First, if the $N$-gon is an equilibrium any radial scaling is also an equilibrium for some different choice of $\Omega$, and so we may assume that each vortex lies on the unit circle. This leaves a single free real parameter $\Omega$, and $z_{1}=e^{2 \pi i / N}, z_{2}=e^{4 \pi i / N}, \ldots, z_{N}=$ 1. We have examined the $N=4$ and $N=6$ (i.e., the marginally stable and the first unstable) cases in detail above. Notice that by abusing notation, we can also consider the dipole as an example of this type with $N=2$, which is stable at the particle level in accordance with the earlier analysis e.g. of [33]. 
Few-Particle Vortex Cluster Equilibria in Bose-Einstein Condensates: Existence and Stability10

The first proposition relies on the following two lemmas.

Lemma 4.2. If $N$ is odd, the $N$-gon is not a fixed point. If $N$ is even, the $N$-gon is a fixed point.

Proof. This is easiest to see in polar coordinates. First assume that the point vortices lie on the unit circle. When $N$ is even, note that by symmetry $\dot{r}_{j}=0$ for each $j$. For $\mathrm{r}=1$, one finds

$$
\begin{aligned}
\dot{\theta}_{j} & =(-1)^{j+1} \Omega+\frac{1}{2} \sum_{k \neq j}(-1)^{k+1} \\
& =\Omega-\frac{1}{2}, \text { if } j \text { is odd } \\
& =-\Omega+\frac{1}{2}, \text { if } j \text { is even }
\end{aligned}
$$

and so the $N$-gon is a fixed point when $\Omega=1 / 2$. On the other hand, when $N$ is odd we have

$$
\begin{aligned}
\dot{\theta}_{j} & =(-1)^{j+1} \Omega+\frac{1}{2} \sum_{k \neq j}(-1)^{k+1} \\
& =\Omega, \text { if } j \text { is odd } \\
& =-\Omega+1, \text { if } j \text { is even }
\end{aligned}
$$

and so for no value of $\Omega$ is the odd $N$-gon a fixed point.

Lemma 4.3. When the $N$-gon is a fixed point, it is unstable for $N \geq 6$.

Proof. This result will require a detailed examination of the corresponding stability matrix. We again consider the system in polar coordinates. The resulting matrix, denoted by $M$, of the linearization is a $2 N \times 2 N$ block matrix made up of $N \times N$ blocks

$$
M:=\left(\begin{array}{cc}
A & B \\
C & D
\end{array}\right)
$$

We compute each of these blocks explicitly. Let $a_{j, k}$ denote the entry of $A$ in the $j^{\text {th }}$ row and $k^{\text {th }}$ column, and similarly define $b_{j, k}, c_{j, k}$ and $d_{j, k}$. Let $\theta_{j k}=\theta_{j}-\theta_{k}$ and $r_{j k}=\left|z_{j}-z_{k}\right|$. Then

$$
\begin{aligned}
& a_{j, k}=\frac{S_{j}\left(r_{k}^{2}-r_{j}^{2}\right) \sin \left(\theta_{j k}\right)}{r_{j k}^{4}}, j \neq k \\
& a_{j, j}=\sum_{k \neq j} \frac{2 r_{k} S_{k}\left(r_{j}-r_{k} \cos \left(\theta_{j k}\right)\right) \sin \left(\theta_{j k}\right)}{r_{j k}^{4}} .
\end{aligned}
$$

In the case of the $N$-gon where $S_{k}=(-1)^{k+1}$ and $r_{j}=1$ for all $j$ we see that the above entries are identically zero, hence $A=\mathbf{0}$, the zero matrix. Next,

$$
b_{j, k}=\frac{r_{j} S_{j}\left(\left(r_{j}^{2}+r_{k}^{2}\right) \cos \left(\theta_{j k}\right)-2 r_{j} r_{k}\right)}{r_{j k}^{4}}, j \neq k
$$


Few-Particle Vortex Cluster Equilibria in Bose-Einstein Condensates: Existence and Stability11

$$
b_{j, j}=\sum_{k \neq j} \frac{r_{k} S_{k}\left(2 r_{k} r_{j}-\left(r_{j}^{2}+r_{k}^{2}\right) \cos \left(\theta_{j k}\right)\right)}{r_{j k}^{4}} .
$$

In this case, when all radii are set to one we find

$$
\begin{aligned}
b_{j, k} & =-\frac{S_{j}}{r_{j k}^{2}} \\
b_{j, j} & =\sum_{k \neq j} \frac{S_{k}}{r_{j k}^{2}} .
\end{aligned}
$$

The matrix $C$ is

$$
\begin{aligned}
c_{j, k} & =\frac{S_{k}\left(\left(r_{j}^{2}+r_{k}^{2}\right) \cos \left(\theta_{j k}\right)-2 r_{j} r_{k}\right)}{r_{k} r_{j k}^{4}}, j \neq k \\
c_{j, j} & =-\sum_{k \neq j} \frac{S_{k}\left(r_{j}^{2}-2 r_{j} r_{k} \cos \left(\theta_{j k}\right)+r_{k}^{2} \cos \left(2 \theta_{j k}\right)\right)}{r_{j} r_{j k}^{4}}-\sum_{k \neq j} \frac{S_{k}\left(r_{j}-r_{k} \cos \left(\theta_{j k}\right)\right)}{r_{j}^{2} r_{j k}^{2}} .
\end{aligned}
$$

Again setting $\mathbf{r}=1$ one obtains after some simplification

$$
\begin{aligned}
& c_{j, k}=-\frac{S_{j}}{r_{j k}^{2}} \\
& c_{j, j}=\sum_{k \neq j} \frac{S_{k}}{r_{j k}^{2}}-\sum_{k \neq j} S_{k} .
\end{aligned}
$$

Here we have used that $r_{j k}^{2}=2-2 \cos \left(\theta_{j k}\right)$ when $\mathbf{r}=\mathbf{1}$. Finally, we have

$$
\begin{aligned}
& d_{j, k}=\frac{S_{j} r_{j}\left(r_{j}^{2}-r_{k}^{2}\right) \sin \left(\theta_{j k}\right)}{r_{k} r_{j k}^{4}}, j \neq k \\
& d_{j, j}=\sum_{k \neq j} \frac{S_{k} r_{k}\left(r_{k}^{2}-r_{j}^{2}\right) \sin \left(\theta_{j k}\right)}{r_{j} r_{j k}^{4}}
\end{aligned}
$$

which is the zero matrix when $\mathbf{r}=\mathbf{1}$. We are interested in the determinant of the matrix

$$
\left(\begin{array}{cc}
-\lambda I & B \\
C & -\lambda I
\end{array}\right)
$$

Since the two matrices in the bottom row of $M$ commute, one has the formula

$$
\operatorname{det}(M)=\operatorname{det}(A D-B C)
$$

and

$$
\operatorname{det}\left(M-\lambda I_{2 N \times 2 N}\right)=\operatorname{det}\left(\lambda^{2} I_{N \times N}-B C\right) .
$$

When $\Omega=\frac{1}{2}, B C$ is a circulant matrix and hence its eigenvalues are real. Therefore, $\lambda$ must be real or purely imaginary. To determine the eigenvalues of a circulant matrix, 
Few-Particle Vortex Cluster Equilibria in Bose-Einstein Condensates: Existence and Stability 12

it is sufficient to know the first row of the matrix. Let $\widetilde{B}=B C$. Then

$$
\begin{aligned}
& \tilde{b}_{1,1}=\left(\sum_{k \neq 1} \frac{S_{k}}{r_{1 k}^{2}}\right)^{2}-\sum_{k \neq 1} S_{k} \sum_{k \neq 1} \frac{S_{k}}{r_{1 k}^{2}}+\sum_{k \neq 1} \frac{S_{1} S_{k}}{r_{1 k}^{4}} \\
& \tilde{b}_{1,2}=\left(\frac{S_{1}}{r_{12}^{2}}\right)\left[\sum_{k \neq 1} \frac{S_{k}}{r_{1 k}^{2}}+\sum_{k \neq 2} \frac{S_{k}}{r_{2 k}^{2}}-\sum_{k \neq 2} S_{k}\right]+\sum_{k \neq 1,2} \frac{S_{1} S_{k}}{r_{1 k}^{2} r_{2 k}^{2}} \\
& \tilde{b}_{1,3}=\left(-\frac{S_{1}}{r_{13}^{2}}\right)\left[\sum_{k \neq 1} \frac{S_{k}}{r_{1 k}^{2}}+\sum_{k \neq 3} \frac{S_{k}}{r_{3 k}^{2}}-\sum_{k \neq 3} S_{k}\right]+\sum_{k \neq 1,3} \frac{S_{1} S_{k}}{r_{1 k}^{2} r_{3 k}^{2}}
\end{aligned}
$$

and in general for $m \neq 1$

$$
\left.\tilde{b}_{1, m}=(-1)^{m+1} \frac{S_{1}}{r_{1 m}^{2}}\left[\sum_{k \neq 1} \frac{S_{k}}{r_{1 k}^{2}}+\sum_{k \neq m} \frac{S_{k}}{r_{m k}^{2}}-\sum_{k \neq m} S_{k}\right]+\sum_{k \neq 1, m} \frac{S_{k}}{r_{1 k}^{2} r_{m k}^{2}}\right)
$$

When $S_{k}=(-1)^{k+1}$, we have

$$
\begin{aligned}
& \tilde{b}_{1,1}=\left(\sum_{k \neq 1} \frac{(-1)^{k+1}}{r_{1 k}^{2}}\right)^{2}+\sum_{k \neq 1} \frac{(-1)^{k+1}}{r_{1 k}^{2}}+\sum_{k \neq 1} \frac{(-1)^{k+1}}{r_{1 k}^{4}} \\
& \tilde{b}_{1, m}=(-1)^{m+1} \frac{1}{r_{1 m}^{2}}\left[\sum_{k \neq 1} \frac{(-1)^{k+1}}{r_{1 k}^{2}}+\sum_{k \neq m} \frac{(-1)^{k+1}}{r_{m k}^{2}}+(-1)^{m+1}\right]+\sum_{k \neq 1, m} \frac{(-1)^{k+1}}{r_{1 k}^{2} r_{m k}^{2}} .
\end{aligned}
$$

The eigenvalues of $\widetilde{B}$ are given by

$$
\gamma_{j}=\sum_{k=1}^{N} \tilde{b}_{1, k} \omega_{j}^{k-1}, j=1, \ldots, N
$$

and the eigenvectors are

$$
v_{j}=\left\{1, \omega_{j}, \omega_{j}^{2}, \ldots, \omega_{j}^{N-1}\right\}
$$

where $\omega_{j}=e^{2 \pi i j / N}$ is the $j^{t h}$ root of unity.

In particular, we find that for chosen even $N$ we can explicitly compute any eigenvalue although a simplified expression for the eigenvalues was not found. For $j=N-2, \gamma_{j}$, the eigenvalues of $\tilde{B}$, will be nonnegative when $N \geq 4$. Using Mathematica, we explicitly compute $\gamma_{N-2}=\left(\frac{\sqrt{2}}{4} N-\sqrt{2}\right)^{2} \geq 0$. Thus $\lambda_{j}= \pm \sqrt{\gamma_{j}}$ is real and nonzero when $N>4$, and the corresponding $N$-gon is unstable. By the same token, the $N$-gon with $N=4$ will be marginally stable.

We point out here that these results, as well as numerical results both at the level of the particle equations and at that of the underlying PDE 32] suggest the following.

Conjecture 4.4. For $N \geq 6$, the $N$-gon has $\frac{N}{2}-2$ distinct pairs of real eigenvalues. 
Few-Particle Vortex Cluster Equilibria in Bose-Einstein Condensates: Existence and Stability 13

At the ODE level, this stems from direct observations for $N=2, N=4, N=6$ and $N=8$, while at the PDE, the relevant observation is that each higher order vortex polygon (for even $N \geq 6$ ) emerges from subsequent supercritical pitchfork bifurcations of the unstable ring dark soliton 42 and hence each additional destabilization adds a real eigenvalue pair to the linear spectrum 32] [see Fig. 5, top right and bottom left, p. 1454]. The $N=4$ state on the other hand emerges as a spectrally stable one from the linear limit. Notice, however, that the stability identified here arises at the large $\mu$ limit. For intermediate values of $\mu$ (i.e., for an intermediate interval thereof) instabilities of oscillatory type may arise due to the PDE nature of the system, i.e., due to collisions of some of the internal modes of the vortex particle system with those of its host background.

\subsection{The Collinear Fixed Point}

We now briefly discuss the generalization of the features of the collinear fixed point that we have numerically observed for larger numbers of vortices.

Conjecture 4.5. For all $N$, there exists a symmetric, collinear fixed point of the equations of motion for alternating sign vortex configurations. For even $N$ such configurations are symmetrically placed around the origin i.e., $( \pm a, 0),( \pm b, 0),( \pm c, 0)$ ..., while for odd $N$ they have the same structure plus an additional vortex placed at the origin.

The existence of such a configuration for any $N$, suggests that there are always real numbers $x_{1}, x_{2}, \ldots, x_{N}$ such that for every $j=1, \ldots, N, \sum_{k=1}^{N}(-1)^{k-j} /\left(x_{j}\left(x_{k}-x_{j}\right)\right)=$ 1 where in the summation, it is implied that $k \neq j$. In the case of $N$ even, the statement is precisely that (and the solution, as our numerical results indicate, has the vortices symmetrically placed around 0 , i.e., $\left.-x_{N / 2},-x_{(N-2) / 2}, \ldots,-x_{1}, x_{1}, \ldots, x_{(N-2) / 2}, x_{N / 2}\right)$. In the case of $\mathrm{N}$ odd, one of the vortices is always placed at 0 , so one can rephrase the statement as: $\sum_{k=1}^{N}(-1)^{k-j} /\left(x_{k}-x_{j}\right)=x_{j}$. The remaining vortices are placed symmetrically around 0 as above. It is clear that this is essentially an algebraic/number-theoretic problem. Effectively, this can be rewritten as a set of $\mathrm{N}$ polynomial equations in $N$ unknonwns. As such, it defines a variety, and the question is whether this variety always has a real point $\left(x_{1}, \ldots, x_{N}\right)$ 43.

In addition, we can generalize our conclusions for the stability of the collinear vortex state as follows.

Conjecture 4.6. The collinear fixed point is unstable for $N \geq 3$ with $N-2$ real directions of instability.

Earlier studies have explored the vortex dipole of $N=2$ at the particle 33. and PDE 30 level. Here, we have studied in a numerically assisted way the cases of $N=3, \ldots, 6$ at the ODE level and such small $N$ cases have also been considered at the PDE level [30, 32, all corroborating the above conclusions. Moreover, at the PDE level, the supercritical pitchfork bifurcation of the rectilinear dark soliton state emanating at each subsequent order a higher collinear vortex configuration clearly signals an agreement with the above statement. Nevertheless, the nature of the corresponding stability matrix is not circulant and we are presently unable to prove such a statement in its full generality although our numerical computations (even with higher $N$ ) fully confirm it. 
Few-Particle Vortex Cluster Equilibria in Bose-Einstein Condensates: Existence and Stability14

\section{Conclusions and Future Challenges}

In the present work, we revisited the topic of few vortex crystal configurations in twodimensional Bose-Einstein condensates. While earlier studies focused on the PDE approach attempting to infer conclusions for the vortex dynamics from the vicinity of the linear limit 22, 23, 24, 25, 26, 27, 28, 29, 30, 32, our approach here has taken a complementary view whereby the vortices have been examined as interacting particle systems (as was done earlier chiefly for the dipole [33, 34, 35], but also for larger vortex numbers in the case where rotation is present [18] or absent 21] for cocirculating vortices). We have shown this approach to be fairly informative towards an understanding of the configurations that may arise for small vortex numbers $N$ and the identification of their stability characteristics. Moreover, the systematic progression towards higher vortex numbers $N$ has enabled us to extract generalizations of the conclusions obtained for lower vortex numbers. In some cases (e.g. for the nonexistence of $N$-gon's for $N$ odd, or for the stability characteristics of $N$-gons with $N$ even), we have been able to prove relevant conclusions in their full generality. In other cases (as e.g. for the collinear configurations), we have formulated general conjectures that may, in turn, stimulate non-trivial connections with other areas of mathematics such as algebra/number theory. These regard, for instance, the existence of solutions mapping into the existence of a real point of a certain variety and the analysis of the properties of the corresponding near-circulant stability matrices. A deeper crosspollinating view that may address such open questions would certainly be a welcome addition to the literature in the near future.

However, there are additional extensions or generalizations of the questions posed herein that merit future investigation in their own right. On the one hand, here we have restricted (for reasons explained) our attention to the case of counter-rotating vortices that may produce fixed point configurations. However, as recent experiments have naturally argued, it is of particular interest to also explore co-circulating vortex states and especially rigidly rotating examples thereof (where all the vortices rotate with the same angular momentum), rendering the co-rotating frame of reference the right one for seeking stationary states of the system. On the other hand, a natural generalization of the present considerations is that of exploring the dynamics of vortex rings in three-dimensional BECs; see e.g. 3, 44, for relevant reviews. In this context, it is also possible to write ordinary differential equations characterizing the interaction of the rings and their intrinsic translational dynamics as e.g. in [45. However, we have not been able to identify simple ODEs that would describe the motion of such rings in a three-dimensional parabolic trap - a key ingredient for the system of ODEs, as we saw above for the case of vortices. The exploration of such vortex rings as interacting particle systems is emerging as an extremely interesting topic for future work and will be deferred for future publications.

Acknowledgments. We thank C.E. Wayne for discussions at the early stage of this work and R. Carretero-González for numerous iterations (including on numerical results of [32]). We also thank F. Hajir for bringing to our attention algebraic connections of the existence problems formulated herein. PGK is grateful to the IMA for its hospitality during the completion of this work and also acknowledges support from NSF-DMS-0806762, NSF-CMMI-1000337 and the US AFOSR via award FA955012-1-0332, as well as the Binational Science Foundation under grant BSF-2010239. 
Few-Particle Vortex Cluster Equilibria in Bose-Einstein Condensates: Existence and Stability 15

[1] C.J. Pethick and H. Smith, Bose-Einstein condensation in dilute gases, Cambridge University Press (Cambridge, 2002).

[2] L.P. Pitaevskii and S. Stringari, Bose-Einstein Condensation, Oxford University Press (Oxford, 2003).

[3] P.G. Kevrekidis, D.J. Frantzeskakis, R. Carretero-Gonzlez, Emergent Nonlinear Phenomena in BoseEinstein Condensates, Springer-Verlag, Berlin, 2008.

[4] R.J. Donnelly, Quantized Vortices in Helium II, Cambridge University Press, New York, 1991;

[5] A. L. Fetter and A. A. Svidzinsky, J. Phys.: Condens. Matter 13, R135 (2001).

[6] A. L. Fetter, Rev. Mod. Phys. 81, 647 (2009).

[7] P.G. Kevrekidis, R. Carretero-González, D.J. Frantzeskakis and I.G. Kevrekidis, Mod. Phys. Lett. B 18, 1481 (2004).

[8] M. Tsubota, K. Kasamatsu and M. Kobayashi, arXiv:1004.5458

[9] J.E. Williams, M.J. Holland, Nature 401, 568 (1999).

[10] K.W. Madison, F. Chevy, W. Wohlleben, J. Dalibard, Phys. Rev. Lett. 84806 (2000).

[11] R. Onofrio, C. Raman, J.M. Vogels, J.R. Abo-Shaeer, A.P. Chikkatur, W. Ketterle, Phys. Rev. Lett. 85, 2228 (2000).

[12] T.W. Neely, E.C. Samson, A.S. Bradley, M.J. Davis, B.P. Anderson, Phys. Rev. Lett. 104, 160401 (2010).

[13] C. Weiler, T. Neely, D. Scherer, A. Bradley, M. Davis, and B.P. Anderson, Nature 455, 948 (2008).

[14] D.V. Freilich, D.M. Bianchi, A.M. Kaufman, T.K. Langin, D.S. Hall, Science 329, 1182 (2010).

[15] D.R. Scherer, C.N. Weiler, T.W. Neely, B.P. Anderson, Phys. Rev. Lett. 98, 110402 (2010).

[16] A.E. Leanhardt, A. Görlitz, A.P. Chikkatur, D. Kielpinski, Y. Shin, D.E. Pritchard, W. Ketterle, Phys. Rev. Lett. 89, 190403 (2002); Y. Shin, M. Saba, M. Vengalattore, T.A. Pasquini, C. Sanner, A.E. Leanhardt, M. Prentiss, D.E. Pritchard, W. Ketterle, Phys. Rev. Lett. 93, 160406 (2004).

[17] C. Raman, J.R. Abo-Shaeer, J.M. Vogels, K. Xu, W. Ketterle, Phys. Rev. Lett. 87, 210402 (2001).

[18] Y. Castin, R. Dum, Eur. Phys. J. D 7, 399 (1999).

[19] S. Middelkamp, P. J. Torres, P. G. Kevrekidis, D. J. Frantzeskakis, R. Carretero-González, P. Schmelcher, D. V. Freilich, and D. S. Hall, Phys. Rev. A 84, 011605 (2011).

[20] J.A. Seman, E.A.L. Henn, M. Haque, R.F. Shiozaki, E.R.F. Ramos, M. Caracanhas, P. Castilho, C. Castelo Branco, P.E.S. Tavares, F. J. Poveda-Cuevas, G. Roati, K.M.F. Magalhaes, V.S. Bagnato, Phys. Rev. A 82, 033616 (2010).

[21] R. Navarro, R. Carretero-Gonzalez, P.J. Torres, P.G. Kevrekidis, D.J. Frantzeskakis, M.W. Ray, E. Altuntas, D.S. Hall, Phys. Rev. Lett. 110, 225301 (2013).

[22] L.-C. Crasovan, V. Vekslerchik, V.M. Pérez-García, J.P. Torres, D. Mihalache, L. Torner, Phys. Rev. A 68063609 (2003).

[23] M. Möttönen, S.M.M. Virtanen, T. Isoshima, M.M. Salomaa, Phys. Rev. A 71, 033626 (2005).

[24] V. Pietilä, M. Möttönen, T. Isoshima, J.A.M. Huhtamäki, S.M.M. Virtanen, Phys. Rev. A 74, 023603 (2006).

[25] L.C. Crasovan, G. Molina-Terriza, J.P. Torres, Ll. Torner, V.M. Pérez-García, D. Mihalache, Phys. Rev. E 66, 036612 (2002).

[26] G. Molina-Terriza, Ll. Torner, E.M. Wright, J.J. García-Ripoll, V.M. Pérez-García, Opt. Lett. 261601 (2001).

[27] A. Klein, D. Jaksch, Y. Zhang, W. Bao, Phys. Rev. A 76, 043602 (2007).

[28] W. Li, M. Haque, S. Komineas, Phys. Rev. A 77, 053610 (2008).

[29] J. Brand, W.P. Reinhardt, Phys. Rev. A 65, 043612 (2002).

[30] S. Middelkamp, P.G. Kevrekidis, D.J. Frantzeskakis, R. Carretero-González, P. Schmelcher, Phys. Rev. A 82, 013646 (2010).

[31] P. Kuopanportti, J.A.M. Huhtamäki, M. Möttönen, Phys. Rev. A 83, 011603(R) (2011).

[32] S. Middelkamp, P.G. Kevrekidis, D.J. Frantzeskakis, R. Carretero-González, P. Schmelcher, Phys. D 240, 1449 (2011).

[33] P.J. Torres, P. G. Kevrekidis, D. J. Frantzeskakis, R. Carretero-González, P. Schmelcher, and D. S. Hall, Phys. Lett. A 375, 3044 (2011).

[34] P.J. Torres, R. Carretero-González, S. Middelkamp, P. Schmelcher, D.J. Frantzeskakis, P.G. Kevrekidis, Comm. Pure Appl. Anal. 10, 1589 (2011).

[35] J. Stockhofe, S. Middelkamp, P. G. Kevrekidis and P. Schmelcher, EPL 93, 20008 (2011).

[36] S.-M. Chang, W.-W. Lin, T.-C. Lin, Int. J. Bif. Chaos 12, 739 (2002).

[37] S. McEndoo, Th. Busch, Phys. Rev. A 79, 053616 (2009).

[38] H. Aref, P. Newton, M. Stremler, T. Tokieda, D. Vainchtein, Advances in applied Mechanics 39, 
Few-Particle Vortex Cluster Equilibria in Bose-Einstein Condensates: Existence and Stability16

$1(2003)$

[39] P. Newton, The N-vortex problem: analytical techniques, Springer-Verlag (New York, 2001).

[40] Y. Chen, T. Kolokolnikov and D. Zhirov, Collective behavior of large number of vortices in the plane, preprint (to appear in Proc. Roy. Soc. A, 2013).

[41] T. Kapitula, P.G. Kevrekidis, R. Carretero-Gonzlez, Physica D 233, 112 (2007).

[42] G. Theocharis, D.J. Frantzeskakis, P.G. Kevrekidis, B.A. Malomed, Yu.S. Kivshar, Phys. Rev. Lett. 90, 120403 (2003); L.D. Carr, C.W. Clark, Phys. Rev. A 74, 043613 (2006); G. Herring, L.D. Carr, R. Carretero-González, P.G. Kevrekidis, D.J. Frantzeskakis, Phys. Rev. A 77, 023625 (2008).

[43] We thank Farshid Hajir for this very interesting observation.

[44] S. Komineas, Eur. Phys. J. Special Topics 147, 133 (2007).

[45] M. Konstantinov, Phys. Fluids 6, 1752 (1994). 